\title{
Chronic leukaemias in the community
}

\section{Eric Wenlong Li}

Haematology advanced trainee, Nepean and Blue Mountains Local Health District, Sydney

\section{David Yeung}

Haematologist, Royal

Adelaide Hospital

Postdoctoral research

fellow, South Australian

Health and Medical

Research Institute, Adelaide

\section{Stephen Fuller}

Haematologist, Nepean

Hospital, Sydney

Associate professor, Nepean Clinical School, University of Sydney

\section{Keywords}

chronic lymphocytic leukaemia, chronic myeloid leukaemia, drug interactions, patient adherence, targeted therapy, tyrosine kinase inhibitors

Aust Prescr 2020;43:126-30 https://doi.org/10.18773/ austprescr.2020.034

\section{SUMMARY}

Patients with chronic myeloid leukaemia and chronic lymphocytic leukaemia are now predominantly managed in an outpatient setting, with infrequent need for hospital-based therapy.

New targeted oral treatments have transformed survival outcomes. An increasing number of patients now have a life expectancy approaching that of the general population.

Suboptimal drug adherence is common and a key reason for therapy failure and poor clinical outcomes.

The pharmacokinetics of new oral targeted drugs are significantly impacted by drug-drug interactions and an altered gastric $\mathrm{pH}$.

Long-term use of some of the new oral drugs is associated with complications, including cardiovascular events and infections, which can be fatal if not recognised.

\section{Introduction}

The management of haematological malignancies has changed dramatically in the last couple of decades with a better understanding of molecular pathobiology. These changes are seen most dramatically in chronic myeloid leukaemia and chronic lymphocytic leukaemia where cytotoxic chemotherapy is increasingly being replaced by oral targeted therapy such as tyrosine kinase inhibitors (see Table). Consequently, patients require fewer hospital-based treatments and are transitioning to outpatient and community care.

New treatment options have transformed survival outcomes. Before the availability of tyrosine kinase inhibitors, the median survival in chronic myeloid leukaemia was 4-7 years. Now most patients have a life expectancy comparable to the general population.' Similarly, median survival in chronic lymphocytic leukaemia is improving.

With increasing prevalence as a result of increased survival rates, it is now common for GPs and pharmacists to be involved in the management of patients receiving oral therapies for chronic leukaemias. It is therefore important to be aware of these new treatments and their potential complications, including significant adverse effects and drug interactions (see Table).

\section{Chronic myeloid leukaemia}

Patients with chronic myeloid leukaemia are commonly asymptomatic and diagnosed during a routine examination or investigation. If symptoms do occur, they are usually related to anaemia, splenomegaly and constitutional symptoms. These symptoms include malaise, lethargy, early satiety, weight loss and abdominal fullness or pain in the left upper quadrant. In peripheral blood, suggestive findings include a persistent neutrophilia often seen with myelocytes, metamyelocytes or occasional blasts (features known as 'left shift'). Basophilia, eosinophilia and thrombocytosis can also be seen.

\section{Diagnosis}

The diagnosis of chronic myeloid leukaemia is established by the detection of the BCR-ABL1 fusion gene (also known as the Philadelphia chromosome) formed by a reciprocal translocation of chromosomes 9 and 22. The protein produced from this fusion gene is a constitutively active, oncogenic tyrosine kinase which drives malignant proliferation.

The most sensitive method for detecting the BCR-ABL1 fusion gene is using a reverse transcriptase-polymerase chain reaction (RT-PCR) test of blood or bone marrow samples. The same test is used to quantify residual disease and monitor treatment response.

\section{Treatment and monitoring}

Chronic myeloid leukaemia presents in three phases chronic, accelerated and blast phase. If left untreated, the chronic phase progresses to blast phase, which is usually fatal. About $90-95 \%$ of patients are diagnosed in the chronic phase. The mainstay of treatment is tyrosine kinase inhibitors, which block the activity of the oncogenic BCR-ABL1 tyrosine kinase. Blastphase disease, which is much less common, may still require high-dose chemotherapy and allogeneic stem cell transplantation.

Tyrosine kinase inhibitors are started at diagnosis and for many patients will be continued for life 
Table Targeted oral therapy for chronic leukaemias - key management points

\begin{tabular}{|c|c|c|c|}
\hline Indication & Drug & Dosing advice & Complications \\
\hline \multirow[t]{3}{*}{$\begin{array}{l}\text { Chronic myeloid } \\
\text { leukaemia }\end{array}$} & Imatinib & $\begin{array}{l}\text { Dose is usually } 400-600 \mathrm{mg} \\
\text { daily. Tablets should be taken with } \\
\text { food to minimise gastrointestinal } \\
\text { adverse effects. }\end{array}$ & $\begin{array}{l}\text { - Compared to other tyrosine kinase inhibitors, imatinib has a higher incidence of nausea, vomiting, } \\
\text { diarrhoea and fluid retention (peripheral oedema, eyelid and periorbital oedema). }\end{array}$ \\
\hline & Dasatinib & $\begin{array}{l}\text { Initial dose is } 100 \text { mg daily. May be } \\
\text { taken with or without food. }\end{array}$ & $\begin{array}{l}\text { - May prolong QT interval, and concomitant drugs that also prolong QT interval should be avoided. } \\
\text { - Significant interaction with antacids taken within } 2 \text { hours of administration. } \mathrm{H}_{2} \text { antagonists and proton } \\
\text { - Rump inhibitors decrease absorption so their combined use with dasatinib is not recommended. } \\
\text { percutaneous drainage. } \\
\text { - Pulmonary hypertension is a rare complication which can be fatal if undetected and requires } \\
\text { immediate cessation of the drug. It may manifest as unexplained dyspnoea or signs of right heart failure. } \\
\text { A transthoracic echocardiogram may find signs of right heart pressures and a right heart catheterisation } \\
\text { is required for a definitive diagnosis. }\end{array}$ \\
\hline & Nilotinib & $\begin{array}{l}\text { Standard dose is } 300 \mathrm{mg} \text { ( } 2 \text { tablets) } \\
\text { twice daily. Must be taken on an } \\
\text { empty stomach ( } 2 \text { hours before, or } \\
1 \text { hour after food) as drug absorption } \\
\text { increases with a high-fat meal. }\end{array}$ & $\begin{array}{l}\text { - May prolong QT interval, and concomitant drugs that also prolong QT interval should be avoided. } \\
\text { - Increased risk of vaso-occlusive vascular events such as ischaemic heart disease, ischaemic } \\
\text { cerebrovascular events and peripheral artery occlusive disease. This warrants proactive management } \\
\text { of cardiovascular risk factors. } \\
\text { - Elevation of blood lipids and glucose has been observed and close monitoring is recommended. } \\
\text { - Increased incidence of pancreatitis. }\end{array}$ \\
\hline \multirow[t]{2}{*}{$\begin{array}{l}\text { Chronic lymphocytic } \\
\text { leukaemia }\end{array}$} & Ibrutinib & $\begin{array}{l}\text { Starting dose is } 420 \mathrm{mg} \text { daily. May be } \\
\text { taken with or without food. }\end{array}$ & $\begin{array}{l}\text { - Use of cytochrome P450 3A4 inhibitors such as azole antifungals and macrolide antibiotics should be } \\
\text { avoided. Dose reduction of ibrutinib may be required (in consultation with the haematologist). } \\
\text { - Significant haemorrhagic complications have been reported and are related to ibrutinib's effect on } \\
\text { platelet activation. Thoroughly assess risk and benefit before concomitant use with anticoagulants } \\
\text { or antiplatelets. } \\
\text { - In patients having surgery with a high risk of bleeding, withhold ibrutinib for 3-7 days before and after } \\
\text { the procedure. } \\
\text { - Ibrutinib use is associated with increased cardiac arrhythmias particularly atrial fibrillation. Management } \\
\text { can be challenging given that concurrent anticoagulation should be avoided. }\end{array}$ \\
\hline & $\begin{array}{l}\text { Venetoclax (initially } \\
\text { used in combination } \\
\text { with rituximab) }\end{array}$ & $\begin{array}{l}\text { Starting dose is } 20 \text { mg titrated weekly } \\
\text { to } 400 \text { mg with monitoring. } \\
\text { Should be taken with food at the } \\
\text { same time each day to ensure } \\
\text { consistent bioavailability as meals } \\
\text { increase bioavailability of venetoclax } \\
\text { 3-5-fold depending on fat content. }\end{array}$ & $\begin{array}{l}\text { - Specialist monitoring required during titration because of the risk of severe tumour lysis syndrome. } \\
\text { Venetoclax is associated with cytopenias, particularly severe neutropenia, which occasionally require } \\
\text { dose modifications. }\end{array}$ \\
\hline
\end{tabular}

etoclax (initially sed in combination Should be taken with food at the each day to ensure 
unless there are unacceptable adverse effects or drug resistance. Treatment response is monitored by quantitative RT-PCR of BCR-ABL1. The median population value of this test is set at $100 \%$ at diagnosis, down to $0.001 \%$ in response to treatment. Some patients who achieve durable and deep molecular remission over years may successfully cease their drug without relapse of disease. This is known as treatment-free remission. However, they must meet stringent criteria and undergo frequent monitoring by their haematologist as rapid relapses can occur.

The choice of tyrosine kinase inhibitor is dependent on disease risk, patient comorbidities and patient preference. There are currently drugs approved for first-line use in Australia - imatinib which is a firstgeneration tyrosine kinase inhibitor, and dasatinib and nilotinib which are both second generation. The rate of disease progression is slightly higher with imatinib, although this drug may be less likely to cause lifethreatening adverse events. Dasatinib and nilotinib can induce faster responses. A third-generation tyrosine kinase inhibitor, ponatinib, is reserved for patients resistant to other tyrosine kinase inhibitors. However, it has a higher rate of vascular toxicity including myocardial infarction, cerebrovascular accidents and peripheral vascular disease.

\section{Chronic lymphocytic leukaemia}

Most patients with chronic lymphocytic leukaemia are asymptomatic at diagnosis. There may be constitutional symptoms (night sweats, weight loss and fever), lymphadenopathy, splenomegaly or both. Occasionally, the disease can be associated with autoimmune haemolytic anaemia, immune thrombocytopenia and recurrent infections. Unexplained and persistent lymphocytosis along with typical blood film findings, such as smudge cells, can suggest chronic lymphocytic leukaemia but do not exclude other lymphoproliferative neoplasms.

\section{Diagnosis}

Diagnosis is based on the presence of a clonal population of lymphocytes ( $\geq 5 \times 10^{9} / L$ for $\geq 3$ months) with an immunophenotype typical of chronic lymphocytic leukaemia (by flow cytometry). A clonal population of less than $5 \times 10^{9} / \mathrm{L}$ is termed monoclonal $\mathrm{B}$-Iymphocytosis, which may progress to chronic lymphocytic leukaemia at a rate of 1-2\% per year.

\section{Treatment and monitoring}

In the early stages, patients without active or symptomatic disease such as progressive cytopenias or constitutional symptoms (unexplained fever, night sweats, weight loss and disabling lethargy) can be monitored without treatment, as there is no survival benefit for early intervention. ${ }^{3}$ In fit patients chemotherapy remains the standard of care (fludarabine, cyclophosphamide and rituximab) with over $80 \%$ of patients having a partial or complete response. ${ }^{4}$

In relapsed or resistant disease, oral targeted therapies commonly used include ibrutinib and venetoclax. Both have high efficacy, particularly in disease carrying high-risk genetic abnormalities such as a TP53 mutation. Ibrutinib is an inhibitor of Bruton's tyrosine kinase, which B-cells rely on for survival and proliferation. In contrast, venetoclax blocks the function of the $\mathrm{Bcl}-2$ protein which protects tumour cells from cell death. ${ }^{4,5}$

\section{Adherence to treatment}

Adherence is critical in ensuring the effectiveness of therapy and GPs can play an important role in checking that patients have been taking their medicines as directed. ${ }^{6}$ In chronic myeloid leukaemia, patients taking imatinib are over 17 times more likely to lose control of the disease when adherence rates are less than 85\%.7 Similarly, studies of ibrutinib for chronic lymphocytic leukaemia have found that dose reduction or significant dose interruptions are associated with worse rates of progression-free survival. ${ }^{8}$

In general, adherence to oral cancer therapy varies markedly (46-100\%) and tends to deteriorate over time. Adherence rates can be influenced by patient, disease, healthcare provider and treatment-related factors. ${ }^{9}$ In chronic myeloid leukaemia, a systematic review noted that drug-related adverse events were the most common reason for intentional nonadherence. Forgetfulness was the most common unintentional reason. ${ }^{10}$

\section{Avoiding drug interactions}

The absorption of these oral targeted drugs is variably affected by alterations in the gastric environment, therefore strict dietary precautions and avoidance of concomitant drugs that increase gastric $\mathrm{pH}$ are important. All drugs mentioned in the Table are major substrates of cytochrome P450 (CYP) 3A4 (in addition to other CYP and non-CYP pathways). Drugs that inhibit or induce CYP3A4 can therefore significantly increase toxicity or reduce efficacy." Prescribers and pharmacists should be vigilant in monitoring and checking for drug interactions (see Table).

\section{Cardiovascular implications}

GPs play an important role in cardiovascular risk management. They can assist in monitoring and managing risk factors such as smoking, hypertension, dyslipidaemia and obesity. 
As the life expectancy of patients with chronic myeloid leukaemia has increased, it is important to address other causes of morbidity. In particular, dasatinib and nilotinib increase the risk of cardiovascular events, such as ischaemic heart disease and cerebrovascular disease.

Hypertension can occur or worsen during treatment with ibrutinib, and antihypertensives should be started or adjusted accordingly. ${ }^{12}$

\section{Infection and vaccination}

Chronic lymphocytic leukaemia can be associated with impaired immunity and an increased risk of infections, particularly in advanced disease and following treatment with immunosuppressive therapy. ${ }^{13}$ International guidelines recommend inactivated influenza vaccine (annually) and pneumococcal vaccine before treatment with B-celldepleting drugs such as rituximab and ibrutinib. 13,14 Vaccination with live vaccines such as varicella zoster is contraindicated in patients with chronic lymphocytic leukaemia as deaths have occurred.12 Selected patients with secondary hypogammaglobulinaemia and recurrent severe infections despite antibiotics may be considered for intravenous immunoglobulin therapy.

Currently, there is no recognised link between chronic myeloid leukaemia and an increased risk of infections, aside from hepatitis $B$ reactivation in those with chronic infection. Antiviral prophylaxis may therefore be appropriate, especially in those with positive hepatitis B serology. ${ }^{15}$ There are limited data on the effectiveness of vaccinations in chronic myeloid leukaemia, although influenza and pneumococcal vaccines should be considered. ${ }^{13}$ Due to limited data, recent international guidelines advise against live attenuated vaccines in patients with chronic myeloid leukaemia who are taking tyrosine kinase inhibitors. ${ }^{14}$

\section{Pregnancy}

Men and women with chronic leukaemia planning to have children should discuss their intentions with their treating haematologist to assess the risks and benefits of ongoing therapy. After careful discussions, treatment may be paused during pregnancy planning through to postpartum. All drugs listed in the Table have caused or are suspected of causing fetal harm (pregnancy category $\mathrm{C}$ or $\mathrm{D}$ ), and their effects on male fertility are uncertain. Outside of specialist advice, effective contraception is generally recommended.

\section{Future directions}

In chronic myeloid leukaemia, current research is focused on deepening the molecular response and management of resistant disease, which includes novel treatments such as asciminib which targets an alternative site on the BCR-ABL1 oncoprotein. Research is also focused on a better understanding of drug pharmacokinetics for personalised dosing

In chronic lymphocytic leukaemia, treatment options are continuing to evolve as long-term remission is now a distinct possibility. Recent trials have found that ibrutinib and venetoclax alone, or in combination, are highly effective in the front-line setting. Newer generation Bruton's tyrosine kinase inhibitors such as acalabrutinib, and therapeutics targeting other signalling pathways such as phosphatidylinositol 3-kinase, have proven to be effective. ${ }^{16}$

\section{Conclusion}

As patients with common chronic leukaemias benefit from rapidly advancing therapies, management priorities are shifting from inducing remission to ensuring a sustained response and managing the complications of treatment. As these patients are increasingly managed in the community, a combined effort between community health professionals and treating specialists is required for optimal outcomes. $<$

David Yeung receives research support from Bristol Myers Squibb and Novartis, and honoraria from Bristol Myers Squibb, Novartis, Pfizer and Amgen. None of these companies has had a role in the preparation of this manuscript.

\section{REFERENCES}

1. Apperley JF. Chronic myeloid leukaemia. Lancet 2015;385:1447-59. https://doi.org/10.1016/S0140-6736(13)62120-0

2. Salem AH, Agarwal SK, Dunbar M, Nuthalapati S, Chien D, Freise KJ, et al. Effect of low- and high-fat meals on the pharmacokinetics of venetoclax, a selective first-in-class BCL-2 inhibitor. J Clin Pharmacol 2016;56:1355-61. https://doi.org/10.1002/jcph.741

3. Hallek M. Chronic lymphocytic leukemia: 2020 update on diagnosis, risk stratification and treatment. Am J Hemato 2019:94:1266-87. https://doi.org/10.1002/ajh.25595

4. Sylvan SE, Asklid A, Johansson H, Klintman J, Bjellvi J, Tolvgård S, et al. First-line therapy in chronic lymphocytic leukemia: a Swedish nation-wide real-world study on 1053 consecutive patients treated between 2007 and 2013 Haematologica 2019;104:797-804. https://doi.org/10.3324/ haematol.2018.200204
5. Seymour JF, Ma S, Brander DM, Choi MY, Barrientos J, Davids MS, et al. Venetoclax plus rituximab in relapsed or refractory chronic lymphocytic leukaemia: a phase $1 \mathrm{~b}$ study. Lancet Oncol 2017;18:230-40. https://doi.org/10.1016/ S1470-2045(17)30012-8

6. Usherwood T. Encouraging adherence to long-term medication. Aust Prescr 2017:40:147-50. https://doi.org/ 10.18773/austprescr.2017.050

7. Ibrahim AR, Eliasson L, Apperley JF, Milojkovic D, Bua M, Szydlo $\mathrm{R}$, et al. Poor adherence is the main reason for loss of CCyR and imatinib failure for chronic myeloid leukemia patients on long-term therapy. Blood 2011;117:3733-6. https://doi.org/10.1182/blood-2010-10-309807 
8. Barr PM, Brown JR, Hillmen P, O'Brien S, Barrientos JC, Reddy NM, et al. Impact of ibrutinib dose adherence on therapeutic efficacy in patients with previously treated CLL/SLL. Blood 2017;129:2612-5. https://doi.org/10.1182/ blood-2016-12-737346

9. Greer JA, Amoyal N, Nisotel L, Fishbein JN, MacDonald J, Stagl J, et al. A systematic review of adherence to oral antineoplastic therapies. Oncologist 2016;21:354-76. https://doi.org/10.1634/theoncologist.2015-0405

10. Noens L, Hensen M, Kucmin-Bemelmans I, Lofgren C Gilloteau I, Vrijens B. Measurement of adherence to BCR-ABL inhibitor therapy in chronic myeloid leukemia: current situation and future challenges. Haematologica 2014;99:437-47. https://doi.org/10.3324/haematol.2012.082511

11. Haouala A, Widmer N, Duchosal MA, Montemurro M, Buclin T, Decosterd LA. Drug interactions with the tyrosine kinase inhibitors imatinib, dasatinib, and nilotinib. Blood 2011;117:e75-87. https://doi.org/10.1182/blood-2010-07-294330

12. Dickerson T, Wiczer T, Waller A, Philippon J, Porter K, Haddad D, et al. Hypertension and incident cardiovascular events following ibrutinib initiation. Blood 2019;134:1919-28. https://doi.org/10.1182/blood.2019000840
13. Hallek M, Cheson BD, Catovsky D, Caligaris-Cappio F, Dighiero G, Döhner H, et al. iwCLL guidelines for diagnosis, indications for treatment, response assessment, and supportive management of CLL. Blood 2018;131:2745-60. https://doi.org/10.1182/blood-2017-09-806398

14. Mikulska M, Cesaro S, de Lavallade H, Di Blasi R, Einarsdottir S Gallo G, et al.; European Conference on Infections in Leukaemia group. Vaccination of patients with haematological malignancies who did not have transplantations: guidelines from the 2017 European Conference on Infections in Leukaemia (ECIL 7). Lancet Infect Dis 2019;19:e188-99. https://doi.org/10.1016/S1473-3099(18)30601-7

15. Knoll BM, Seiter K. Infections in patients on BCR-ABL tyrosine kinase inhibitor therapy: cases and review of the literature. Infection 2018;46:409-18. https://doi.org/10.1007/ s15010-017-1105-1

16. Sharman JP, Egyed M, Jurczak W, Skarbnik A, Pagel JM, Flinn IW, et al. Acalabrutinib with or without obinutuzumab versus chlorambucil and obinutuzmab for treatmentnaive chronic lymphocytic leukaemia (ELEVATE TN): a randomised, controlled, phase 3 trial. Lancet 2020;395:1278-91. https://doi.org/10.1016/S0140-6736(20)30262-2

\section{FURTHER READING}

Carrington C. Oral targeted therapy for cancer. Aust Prescr 2015;38:171-6. https://doi.org/10.18773/austprescr.2015.060 